\title{
Violencia de género en el ámbito universitario. Medidas para su superación
}

\author{
Consol Aguilar Ródenas \\ UNIVERSIDAD JAUME I \\ María José Alonso Olea \\ Universidad del País VAsco \\ Patricia Melgar Alcatud y Silvia Molina Roldán \\ UNIVERSITAT DE BARCELONA
}

\section{Resumen}

Este artículo expone parte de los resultados de la primera investigación sobre violencia de género en las universidades españolas ${ }^{1}$. A partir de la revisión de investigaciones internacionales, mostramos que la violencia de género tiene una presencia importante en las universidades. Posteriormente, identificamos medidas para la prevención y superación de la violencia de género que ya se están desarrollando en algunas de las universidades de más prestigio internacional. Ambos aspectos son el punto de partida para el estudio de la violencia de género en las universidades españolas y para el diseño de medidas que contribuyan a su superación.

Palabras clave: universidad, violencia de género, agresión sexual, acoso, investigación, medidas, prevención.

\section{Summary}

This article contains some of the results from the first research on gender violence in Spanish universities. Based on a review of international research, we demonstrate that gen- der violence is prevalent in universities. Subsequently, we identify measures to prevent and overcome gender violence which are already being developed in some of the most prestigious international universities. Both of these aspects are the starting off point to study gender violence in Spanish universities and to design measures which contribute towards overcoming it.

KEYWORDS: university, gender violence, sexual assault, harassment, research, measures, prevention.

\section{Introducción}

La violencia de género es una problemática social que afecta a mujeres de diferentes edades, clases sociales, culturas o niveles académicos y que supera estereotipos existentes respecto a quiénes la sufren, por qué y dónde se produce. Como parte de esta realidad, los datos de diversas investigaciones internacionales indican que también es un fenómeno que se da en el ámbito universitario y, como consecuencia, existen ya numerosas iniciativas y experiencias en diversas universidades 
del mundo que implementan medidas para prevenir situaciones de violencia de género en el contexto universitario.

Esta investigación parte de esta realidad, así como de la idea de que en las universidades españolas la violencia de género se manifiesta en diferentes formas, de la misma manera que lo hace en otros ámbitos sociales. Se trata de una realidad que muchas personas conocemos pero sobre la que apenas se ha roto el silencio para sacarla a la luz pública y, por lo tanto, no se están desarrollando acciones que contribuyan a erradicarla.

En este artículo presentamos parte de los resultados obtenidos en el proyecto $\mathrm{I}+\mathrm{D}+\mathrm{I}$ Violencia de género en las universidades españolas financiado por el Instituto de la $\mathrm{Mu}$ jer, Ministerio de Trabajo y Asuntos Sociales (2006-2008) ${ }^{2}$. El objetivo de este proyecto ha sido estudiar la presencia de violencia de género en el contexto universitario español e identificar medidas que puedan contribuir a superarla. Este artículo se centra en los resultados obtenidos acerca de la implementación de medidas contra la violencia de género en el contexto universitario.

Esta investigación parte también del trabajo previo realizado por diferentes investigadoras e investigadores de CREA, Centro Especial de Investigación en Teorías y Prácticas Superadoras de Desigualdades de la Universidad de Barcelona, en el ámbito del género, la socialización preventiva de la violencia de género, los trabajos iniciados por Jesús Gómez sobre modelos de atracción (2004) y de los debates científicos que ha impulsado el Grupo de Mujeres de CREA Safo.

Este trabajo ha sido el segundo que se desarrolla en nuestro país centrada en estudiar la violencia de género específicamente en el contexto universitario. La primera investigación fue realizada en año 2006, (Valls y otros, 2005-2006) pero únicamente abarcaba las universidades catalanas. Ambas investigaciones han sido dirigidas por la Dra. Rosa Valls Carol.

\section{Metodología}

La investigación se ha basado en una metodología de orientación comunicativa crítica (Gómez y otros, 2006). Esta metodología se propone generar conocimiento a través de la creación de un diálogo intersubjetivo y pone énfasis tanto en los componentes que suponen mecanismos de desigualdad, como en los componentes transformadores que favorecen la superación de las desigualdades. En nuestra investigación, este enfoque se ha llevado a cabo combinando técnicas cuantitativas y cualitativas. La orientación comunicativa se ha concretado en la inclusión de personas vinculadas a la temática trabajada en el proyecto y la población objeto de estudio y el diálogo con ellas en diferentes momentos del estudio.

El estudio se ha realizado durante tres años. En su desarrollo, y en lo que respecta a los resultados que discutimos en este artículo, se han llevado a cabo los siguientes análisis.

- Análisis de las investigaciones que, a nivel internacional, se están realizando sobre violencia de género en las universidades. Este análisis ha permitido identificar medidas, políticas universitarias o propuestas implementadas en otras universidades que están contribuyendo a su prevención y superación. Asimismo, en este análisis se han identificando los indicadores más utilizados en el estudio de la violencia de género, que han servido como base para la implementación de una encuesta sobre violencia de género en diferentes universidades españolas con el objetivo de analizar su presencia en el contexto universitario español. La revisión bibliográfica y documental se ha llevado a cabo partiendo de criterios de exhaustividad y cientificidad, recurriendo a las bases de datos de más reconocimiento internacional en Ciencias Sociales y Educativas (ERIC, SOCIOFILE), a las publicaciones periódicas inter- 
nacionales de mayor impacto científico (Journal Citation Reports. Social Science Edition/ISI) y a los proyectos I+D desarrollados en España.

- Elaboración de un inventario de medidas y recursos que existen en diferentes universidades contra la violencia de género y el posterior análisis de buenas prácticas bajo dimensiones como la solidaridad entre mujeres, el apoyo a la víctima y la implicación de la comunidad educativa. Además, a través de la realización de relatos comunicativos de vida cotidiana con estudiantado y entrevistas con profesorado y personal de servicios y administración, hemos conocido la acogida que tendrían estas propuestas en nuestras universidades así como su utilidad y viabilidad a la hora de aplicarlas.

\section{Análisis e interpretación de los resultados}

\subsection{Evidencias acerca de la violencia de género en las universidades. Estado de la cuestión}

Las investigaciones que a nivel internacional describen la presencia de la violencia de género en las universidades nos permiten dibujar este fenómeno a partir de algunas características como las que describimos a continuación:

\subsubsection{La presencia de violencia de género en las universidades}

La mayoría de investigaciones sobre la temática estudiada han sido desarrolladas en Norteamérica. Asimismo, son estos países los que cuentan en la mayoría de sus universidades con medidas de atención a las victimas y de prevención de la violencia de género. En el contexto europeo encontramos pocas investigaciones al respecto; no obstante, algunas universidades, tal y como veremos en el siguiente apartado, implementan medidas en este sentido. En España, la investigación que presentamos en este artículo es la segunda que se realiza, y la primera que se dirige a analizar la problemática de la violencia de género en las universidades en el conjunto de España. Las medidas de atención y prevención son prácticamente inexistentes en la mayoría de nuestras universidades.

En el contexto norteamericano, según Fonow (1992), de las violaciones que suceden en los campus universitarios en los EE UU, el 54\% suceden en las citas; además, el 35\% de los chicos universitarios afirman que cometerían una violación si pudiesen. En este mismo contexto, un estudio que analizó 140 encuestas a mujeres universitarias pertenecientes a una hermandad femenina concluyó que el 51\% habían sufrido al menos un acto de agresión sexual desde que tenían 14 años; de estas mujeres, el 83\% había sufrido al menos una de esas situaciones mientras estaba en la universidad. Un 95\% de las mujeres que habían sufrido esta situación conocían a su agresor, que era su novio, amigo o conocido. En un 38\% de los casos lo habían conocido el mismo día o durante la noche de la agresión. En un 41\% de los casos la violación o intento de agresión tuvo lugar en las casas de hermandades (Copenhaver y otros, 1991).

Más recientemente, el estudio de Gross (Gross y otros, 2006) destaca que, desde su matriculación en la universidad, el 27\% de las 903 mujeres universitarias que participaron en su estudio habían sufrido algún tipo de abuso o situación no deseada, desde besos y caricias hasta relaciones sexuales.

En el contexto de Cataluña, la investigación llevada a cabo (Valls y otros, 2005-2006) ha mostrado que un $58 \%$ de las 368 chicas universitarias que respondieron a la encuesta afirmaban haber sufrido o conocer alguna de las situaciones de violencia de género en la universidad sobre las que se les preguntaba. 


\subsubsection{Las víctimas no identifican situaciones de violencia de género como tales}

Un dato sobre el que hay que estar alerta es que muchas mujeres que sufren este tipo de situaciones no las consideran agresiones sexuales, aun habiendo sido forzadas a tener relaciones sexuales no deseadas, como una violación (Copenhaver y otros, 1991; Kalof, 1993). A esta falta de reconocimiento de la violencia frecuentemente se une la falta de denuncia. La investigación llevada a cabo por Gross es muy significativa en este sentido: de las 246 mujeres entrevistadas que habían sufrido alguna situación de violencia de género, únicamente 4 explicaron el suceso a la policía (Gross y otros, 2006). Este estudio explicita también la existencia de una visión estereotipada de la violación, cuyas consecuencias son, entre otras, la asunción por parte de la víctima de un grado de responsabilidad en la provocación de la situación.

La investigación de Bondurant (2001) destaca que las víctimas que sufren elevados niveles de violencia durante la agresión identifican en mayor medida la situación como una violación. Asimismo, añade otro elemento relevante: en los grupos de amigas en los que se habla abiertamente sobre este tipo de situaciones, las mujeres están más predispuestas a reconocerlas como agresiones sexuales o violaciones. Así, es de gran importancia señalar cómo el diálogo y las interacciones entre iguales pueden ser un factor clave a la hora de identificar situaciones de violencia de género y, por lo tanto, de prevenirlas. Este aspecto será también fundamental tenerlo en cuenta a la hora de profundizar en el análisis de medidas y actuaciones para la prevención de la violencia de género en las universidades.

\subsubsection{Culpabilización de las víctimas}

Un elemento que contribuye a la no identificación de la violencia de género ni al apoyo a las víctimas, es la culpabilización de las mismas. Cowan (2000), analizando las creencias que culpabilizan a las mujeres de la violación y que exculpan a los hombres, encontró en su estudio respuestas que apuntaban ideas como que las "víctimas" son las que provocaron la situación y, por lo tanto, las responsables de que se produjera, que las violaciones sólo son perpetradas por hombres con patologías mentales, o que son situaciones que los hombres que las llevan a cabo no pueden evitar por sus necesidades sexuales. Cowan identifica que este rechazo, insolidaridad y desconfianza entre las propias chicas no son actitudes aisladas, sino que deben analizarse en el contexto de una serie de creencias sociales producto de la socialización que toleran y permiten la violencia y acoso sexuales, contribuyendo a crear un ambiente de hostilidad hacia las mujeres.

\subsubsection{Modelos de relación que favorecen la violencia de género}

Existen investigaciones dirigidas a estudiar las dinámicas asociadas a las relaciones en las que se produce violencia en las citas entre universitarios y universitarias. Charkow y Nelson (2000) analizaron una población de 178 mujeres estudiantes universitarias e identificaron que las relaciones en las que se producía violencia o coacción sexual en las citas respondían a esquemas de relaciones caracterizados por aspectos de dependencia y aceptación del abuso confundiéndolo con amor o con ideales tradicionales románticos. Estos autores destacaron que la intervención con programas de prevención de la violencia en las citas tendría que dirigirse a trabajar los elementos que caracterizan este tipo de relaciones no saludables.

Otra línea de investigación se ha centrado en la relación entre las actitudes de apoyo a las relaciones sexuales forzadas que se da en las fraternidades y la violencia se- 
xual (Yancey, 1989; Copenhaver, 1991; Fonow, 1992; Boeringer, 1999; Boswell y Spade, 1996). Kalof (1993) encontró en su estudio que, en comparación con otras mujeres universitarias, las miembros de hermandades manifestaban actitudes más estereotipadas sobre la aceptación de la violencia interpersonal y la aceptación de mitos sobre la violación, tales como "a las mujeres les gusta que las fuercen para mantener relaciones sexuales", "las chicas agradables no son violadas", o "las víctimas de violación son promiscuas o tienen mala reputación”.

\subsubsection{La universidad como contexto hostil para las mujeres}

Otras investigaciones no se han centrado en identificar situaciones concretas de violencia de género en las universidades sino en analizar la universidad como una institución donde se genera un ambiente adverso hacia las mujeres. Un trabajo canadiense (Osborne, 1995) destaca cómo las universidades son un contexto mucho más desfavorable para las mujeres que para los hombres, partiendo de diferentes contribuciones de estudios previos en los que se analiza cómo el contexto universitario devalúa y margina a la mujer. Así, se considera que el acoso sexual y la misoginia también son formas de violencia contra las mujeres y que pueden manifestarse con normalidad en los currículos académicos, en las discusiones y debates en las aulas universitarias, siendo un mecanismo de subordinación y opresión hacia las mujeres que se vive en el día a día universitario. También se destaca que hay pocos estudios que hayan identificado este tipo de actitudes, comportamientos y prácticas como formas de violencia o que hayan analizado la conexión entre formas de violencia y otros mecanismos de subordinación de las mujeres.

Otro aspecto fundamental, que nos concierne especialmente, es que se denuncia una realidad muy próxima a situaciones que se están viviendo en la actualidad en las universidades españolas: la situación adversa en que encuentran las mujeres que se han atrevido a romper el silencio, denunciando y haciendo públicas las situaciones de violencia de género (Puigvert, 2008; Valls y otros, 2008).

Centrándose en el acoso sexual por parte de profesorado universitario al estudiantado, Kalof, Eby, Matheson y Kroska (2001) obtienen como resultado el destacable dato de que, de las 525 personas encuestadas, el 40\% de las mujeres y el $28,7 \%$ de los hombres habían sido acosadas y acosados por profesorado de la universidad.

Algunas investigaciones ponen de manifiesto que entre las causas por las que muchas de las mujeres que sufren situaciones de violencia de género, incluso agresiones sexuales y violaciones no denuncian, se encuentra el sentimiento de que la institución universitaria no las tomará en serio o no las apoyará (Hensley, 2003). Por otro lado, las estudios también han mostrado que los y las estudiantes creen que los sistemas de gobierno en las universidades no abordan de manera adecuada la violencia de género en el campus y los comportamientos que la potencian, lo que lleva a la apatía entre el alumnado y a que no se informe de incidentes de violencia que han sucedido en el contexto universitario. Es por tanto necesario que las universidades empiecen a cambiar este ambiente potenciando la no tolerancia hacia cualquier tipo de violencia (Bryant y Spencer, 2003).

\subsection{Medidas de prevención y atención de la vio- lencia de género}

La necesidad identificada a través de las investigaciones de implementar medidas de prevención y atención se ve reflejada en la existencia de las mismas contra la violencia de género en las universidades de más prestigio internacional. A través de la revisión de las actuaciones desarrolladas en las 
universidades a nivel internacional hemos podido recoger diferentes tipos de acciones que se deben tomar en cuenta a la hora de desarrollar planes de actuación contra la violencia de género en nuestro contexto, donde este campo aún no está desarrollado. En el presente apartado presentamos una categorización de ejemplos de medidas que actualmente se están aplicando en diferentes universidades.

\subsubsection{Medidas de identificación de las situa- ciones de violencia de género}

Encontramos estas informaciones, en primer lugar, en las páginas web de las universidades (California Institute of Technology, Massachusetts Institute of Technology, Duke University, University College London), donde se dedica algún apartado específico a proporcionar información sobre qué es la violencia de género, a identificar diferentes tipos de violencia y a poner ejemplos que permitan su detección.

Asimismo encontramos trípticos o guías con información en los que se define qué es violencia de género, acoso, agresión y abuso sexual, con ejemplos sobre situaciones concretas, para facilitar su identificación, información sobre servicios a los que acudir si se sufre una de ellas y el posicionamiento como institución en contra de la violencia de género, el acoso y abuso sexual (Princeton University, Harvard University, University of Pennsylvania, University of Manchester, Dartmouth College, University of Cambridge).

Por otro lado, se organizan también conferencias o grupos de discusión para que los alumnos sepan qué es la violencia de género y proporcionarles herramientas para detectarla (Columbia University).

\subsubsection{Servicios de atención y asesoramiento}

En este grupo de medidas encontramos, en primer lugar, el asesoramiento por parte de asociaciones de estudiantes, donde se pueden encontrar actividades como cursos de formación sobre diferentes temáticas, entre ellas la prevención de la violencia de género (Campus-wide Education, Princeton University), información y asesoramiento sobre el abuso de drogas, las enfermedades de transmisión sexual, el acoso y la agresión sexual, así como información sobre los servicios a los que pueden acudir (Health Educators, Yale University), asesoramiento a estudiantado con problemas de stress y que sufren acoso o abusos sexuales (Walden - Anonymous and confidential peer counseling, Yale University), y sensibilización, prevención y apoyo a toda la comunidad universitaria para concienciar sobre el problema de la violencia de género y proponer acciones para acabar con ella (Stop our silence, Massachusetts Institute of Technology Institute Technology).

En segundo lugar, encontramos las oficinas de atención y prevención del acoso y agresión sexual en las universidades. Suelen ser oficinas creadas en las universidades que coordinan los diferentes programas de prevención y atención de las agresiones sexuales y del acoso sexual. Algunos ejemplos son la Office of Sexual Assault Prevention and Response (Harvard University), donde podemos encontrar información y apoyo a los estudiantes que han sufrido cualquier tipo de abuso sexual o relación violenta, acompañamiento para la presentación de informes o para recibir atención médica, información y apoyo a los familiares y amistades de las víctimas, talleres educativos sobre la violencia sexual y otros temas relacionados; el Sexual Violence Advisory Board (Stanford University), comisión formada por estudiantado, profesorado y personal de administración y servicios que tiene como finalidad coordinar medidas de prevención y atención a la violencia sexual en la universidad; y la OMBUDS Office, creada para ofrecer a los miembros de la universidad asistencia confidencial e informal para resolver 
conflictos dentro del campus (California Institute of Technology).

En tercer lugar, encontramos otros servicios de atención a las víctimas de violencia de género en la universidad, como teléfono de urgencias, para asesorar a la persona que ha sufrido una agresión sexual sobre cómo contactar con la policía, el servicio sanitario o el hospital de la universidad (Yale University); teléfonos de asesoramiento con la finalidad, entre otras cosas, de dar información sobre qué hacer en caso de haber sufrido acoso sexual o agresión sexual (Yale University); centros de atención médica (Harvard University), incluso con una sección específica para atender a las víctimas de agresiones sexuales (Massachusetts Institute of Technology MIT); o la comisaría de policía en el campus, (Harvard University, Massachusetts Institute of Technology), algunas de las cuales tienen servicios específicos que atienden los casos de agresión sexual (California Institute of Technology). También existen tutores y tutoras que se pueden asignar a los y las estudiantes que denuncien casos de violencia o abusos para que les informen sobre los recursos que ofrece la universidad y le asesoren en los procesos de denuncia (University of Cambridge), así como servicios gratuitos de terapia psicológica grupal e individual para víctimas de acoso y agresión sexual (Dartmouth College).

\subsubsection{Medidas para facilitar la denuncia y romper el silencio}

Se trata de universidades que disponen de oficinas, grupos y foros de denuncia que sirven para esta finalidad. Existen oficinas donde poder denunciar casos de abuso, que suelen depender de alguna asociación o institución de la universidad (Universidad de Columbia). Los grupos tienen como objetivo concienciar sobre el problema de la violencia de género y explicar en qué consiste; acostumbran a ser grupos de debate, for- mados habitualmente por estudiantado, aunque también los hay dirigidos al profesorado y al personal de administración y servicios (Duke University). Con una finalidad similar, los foros en Internet se utilizan -principalmente en universidades británicas- para que el estudiantado pueda explicar sus experiencias, consultar dudas o discutir sobre temas de género (University College London, University of Manchester).

\subsubsection{Información sobre servicios y lugares donde acudir para buscar asesoramiento}

Esta información va dirigida tanto a víctimas de situaciones de violencia de género como a entidades que quieran organizar actividades de prevención, formación o similares. En el primer caso, encontramos la guía que se da al estudiantado cuando se matricula en la universidad, donde se ofrece información sobre los servicios a los cuales se puede acudir. En esta guía también se encuentra el posicionamiento de la universidad de "Tolerancia Cero" hacia el acoso y cualquier otro tipo de violencia de género (University of Cambridge).

En el segundo caso, encontramos centros dirigidos a las organizaciones universitarias que tratan la violencia de género, cuyo objetivo es reducir los delitos contra las mujeres y mejorar los servicios a las víctimas en la universidad, aplicando programas de educación y prevención y desarrollando la investigación de estrategias que prevengan la violencia de género (University of Pennsylvania, Drexler University, University of the Sciences in Philadelphia).

\subsubsection{Medidas que contribuyen a crear am- bientes no hostiles hacia las mujeres, de "To- lerancia Cero" hacia la violencia de género y de solidaridad hacia la víctima}

Encontramos, en primer lugar, oficinas que se dedican a la resolución de conflictos re- 
lacionados con la violencia de género. Normalmente son oficinas que también recogen denuncias y una vez estudiadas ponen en marcha servicios de mediación entre la víctima y el agresor, entrevistan a las partes, valoran posibles sanciones, etc. También se encargan de identificar problemas sobre la violencia de género y proponer políticas a la universidad para que las lleve a cabo, analizan los programas realizados por la universidad, investigan estrategias que prevengan la violencia de género y mejoran los servicios prestados por la universidad (California Institute of Technology, University of Manchester). En segundo lugar, encontramos los grupos de apoyo, que analizan cuestiones de género, analizan acciones para acabar con la violencia de género, atienden las necesidades de las mujeres que acuden a los grupos y ofrecen información y recursos (University of Wisconsin, Dartmouth College).

\subsubsection{Actividades de prevención y formación}

Encontramos centros de mujeres (Women's Center) donde se desarrollan una serie de actividades y servicios como asesoramiento confidencial, charlas, talleres para evitar, disuadir y resistir la violencia física y verbal, grupos de apoyo, y eventos culturales como proyecciones de películas y tertulias que analizan una serie de cuestiones relacionadas con la mujer y el género (California Institute of Technology). En este marco hallamos también cursos de autodefensa, habitualmente organizados por los departamentos de policía de las universidades, como el Rape Aggression Defense (Universidad de Dartmouth).

Existen, asimismo, talleres de prevención, a través de los cuales se trabajan diversos temas relacionados con la violencia de género, y se proporciona información sobre qué hacer en el caso de sufrir una situación de acoso, abuso o agresión sexual. Por otro lado, toda la comunidad universitaria puede disponer de materiales diversos para trabajar la prevención de la violencia de género como películas, libros y guías (Princeton University, Yale University).

Existen actuaciones dirigidas a los hombres. Por ejemplo, el National Organization of Men's Outreach for Rape Education (NO $M O R E)$ es un grupo de hombres que trabajan para la formación y la educación de los hombres sobre acoso sexual (Yale University), y Men Against Violence es un grupo dedicado a acabar con la violencia de los hombres contra las mujeres, los niños, las niñas u otros hombres, promover espacios de cambio y explorar la diversidad de masculinidades que se dan en la universidad (Columbia University).

\subsubsection{Posicionamiento público como insti- tución universitaria contra las diferentes for- mas de violencia de género y acoso en el ám- bito universitario}

El posicionamiento de las universidades toma forma a través de diferentes acciones. En primer lugar, existen actos de reivindicación contra la violencia de género, que están contenidos en un calendario sobre este tipo de eventos (Harvard University). En segundo lugar, se promueve el rechazo de toda forma de violencia o abuso mediante las políticas de la universidad, que también permiten sancionar este tipo de sucesos (University of Pennsylvania, University College London, University of Manchester, University of Oxford, London School of Economics). En tercer lugar, existen casos en que la universidad o alguna de sus organizaciones condena la violencia de género mediante declaraciones en sus páginas web, en las guías que ofrecen al estudiantado, o a través de otros medios (California Institute of Technology, Duke University, Dartmouth College).

\subsubsection{Publicaciones e informes}

Finalmente, algunas universidades hacen publicaciones e informes sobre acoso sexual 
en la universidad (Harvard University, Dartmouth Collage, Duke University).

\section{Conclusiones}

Nuestra socialización sigue alimentando valores desiguales que desde la infancia crean caminos diferentes y excluyentes para las niñas y los niños, como desde hace ya muchos años denuncia el movimiento feminista. Es cierto que se han realizado avances, especialmente desde los sistemas educativos, pero aún quedan muchas cosas que cambiar porque la socialización no se produce sólo a través de los currículos educativos sino que inciden muchos aspectos, como las formas de relación que vivimos en el entorno familiar, las relaciones con los amigos y las amigas, los medios de comunicación, las películas, los libros y las constantes interacciones en las que tomamos parte (Froufe, 1997; Oliver y Valls, 2004; Valls y otras, 2008).

Esta investigación muestra que esta socialización se refleja también en el contexto universitario y que es necesario implementar medidas para su superación. La existencia de medidas en las universidades contribuye a construir un ambiente donde las agresiones son más fácilmente identificables, se favorece su denuncia y se abre el camino hacia su erradicación. A través de ellas, nuestras universidades pueden crear ambientes de tolerancia cero ante la violencia de género -en general y en concreto la que se da en la institución universitaria- contribuyendo a una socialización preventiva de la violencia de género y rompiendo el silencio en las instituciones universitarias, como ya se ha hecho en otros países y como está haciendo en España la sociedad en general gracias, principalmente, al trabajo de los grupos de mujeres y otros movimientos sociales y de las investigaciones. Cuando las instituciones pongan las medidas para que las mujeres perdamos el miedo a denunciar la violencia de género que sufrimos y cuando no dé miedo hacer investigación sobre estos temas tendremos unas universidades no sólo menos sexistas sino también más científicas y humanas.

\section{Referencias bibliográficas}

Boeringer, S. B. (1999): "Research note: Associations of rape-supportive attitudes with fraternal and athletic participation". Violence Against Women, 5, pp. 81-89.

Bondurant, B. (2001): "University's women acknowledgment of rape". Violence Against Women, 7, pp. 294-314.

Boswell, A.; Spade, J. Z. (1996): "Fraternities and collegiate rape culture: Why are some fraternities more dangerous places for women?". Gender and Society, 10, pp. 133-147.

Bryant, S.; Spencer, G. (2003): “University Students. Attitudes about Attributing Blame in Domestic Violence”. Journal of Family Violence, 18, pp. 369-376.

Charkow, W. B.; Nelson, E. S. (2000): "Relationship dependency, dating violence and scripts of female college students". Journal of College Counseling, 3, pp. 17-28.

Copenhaver, S.; Grauerholz, E. (1991): "Sexual victimization among sorority women: exploring the link between sexual violence and institutional practices". Sex Roles, 24, pp. 31-41.

Cowan, G. (2000): "Women's hostility toward women and rape and sexual harassment myths". Violence Against Women, 6, pp. 238-246.

Fonow, M. M.; Richardson, L.; Wemmerus, V. A. (1992): "Feminist rape education: Does it work?" Gender and Society, 6, pp. 108-121.

Froufe Quintas, S. (1997). "La mujer ante el Tercer Milenio”. Pedagogía Social. Revista Interuniversitaria. 15-16, pp. 87-96.

Gómez, J. (2004): El amor en la sociedad del riesgo. Barcelona: El Roure.

Gómez, J.; Latorre A.; Sánchez M.; Flecha R. (2006): Metodología Comunicativa Crítica. Barcelona: El Roure. 
Gross, A. M.; Winslett, A.; Roberts, M.; Gohm, C. L. (2006): "An examination of sexual violence against college women". Violence Against Women, 12 , pp. 288-300.

Hensley, L. (2003): "Sexual assault prevention programs for college men: An exploratory evaluation of the men against violence model". Journal of College Counseling, 6, pp. 166-176.

Kalof, L. (1993): "Rape-supportive attitudes and sexual victimization experiences of sorority and nonsorority women". Sex Roles, 29, pp. 767-780.

Kalof, L.; Eby, K.; Matheson, J. L.; Kroska, R. J. (2001): "The influence of race and gender on students self-reports of sexual harassment by college professors". Gender and Society, 15, pp. 282-302.

Oliver, E.; Valls, R. (2004): Violencia de género. Investigaciones sobre quiénes, por qué y cómo superarla. Barcelona: El Roure.

Osborne, R. L. (1995): "The continuum of violence against women in Canadian universities. Toward a new understanding of the chilly campus climate". Women's Studies International Forum, 18, pp. 637646.

Puigvert, L. (2008): "Breaking the Silence: The Struggle Against Gender Violence in Universities". International Journal of Critical Pedagogy, 1, pp. 16.

Valls, R. y otros (2005-2006): Violències de gènere a l'àmbit universitari? Realitats, formes i superació. Agència de Gestió d'Ajuts Universitaris i de Recerca. Generalitat de Catalunya.

Valls, R. y otros. (2004-2008). Violencia de género en las universidades españolas. Ministerio de Trabajo y Asuntos Sociales. Instituto de la Mujer. Plan Nacional de I+D+I 2004 - 2007. № Expediente 50/05.

Valls, R.; Flecha, A.; Melgar, P. (2008): "Violència de gènere a les universitats catalanes: mesures per a la prevenció i superació". Temps d'Educació, 35, pp. 201-216.
Valls, R.; Puigvert, L.; Duque, E. (2008). “Gender Violence Among Teenagers: socialization and prevention". Violence Against Women, 14, pp. 759785 .

Yancey, P.; Hummer, R. (1989): "Fraternities and Rape on Campus". Gender and Society, 3, pp. 457473.

\section{Notas}

${ }^{1}$ Las cuatro autoras son miembros del Proyecto Violencia de género en las Universidades españolas) Ministerio de Trabajo y Asuntos SocialesInstituto de la Mujer. Plan Nacional I+D+I. 20052008.

${ }^{2}$ El equipo de investigación del proyecto está integrado por los siguientes miembros: Rosa Valls, María José Alonso Olea, Consol Aguilar Ródenas, Luis Torrego Egido, Pilar Colas Bravo, Lola Frutos, Jesús Gómez, Montse Fisas, María Padrós, Ainhoa Flecha, Miguel Ángel Pulido, Laura Ruiz, Patricia Melgar, Laura López y Silvia Molina.

DiRECCIÓN DE LAS AUTORAS: Consol Aguilar Ródenas. Universidad Jaume I. Facultad de Ciencias Humanas y Sociales. Av. De Vicent Sos Baynat, s/n. 12071 Castelló de la Plana.

Correo electrónico: aguilar@edu.uji.es.

Fecha de recepción del artículo: 21.X.2008

Fecha de aceptación definitiva: 08.I.2009 